\title{
Gantenerumab reduces amyloid- $\beta$ plaques in patients with prodromal to moderate Alzheimer's disease: a PET substudy interim analysis
}

Gregory Klein ${ }^{1 *}$, Paul Delmar ${ }^{2}$, Nicola Voyle ${ }^{3}$, Sunita Rehal ${ }^{3}$, Carsten Hofmann ${ }^{1}$, Danielle Abi-Saab², Mirjana Andjelkovic ${ }^{2}$, Smiljana Ristic², Guoqiao Wang ${ }^{4}$, Randall Bateman ${ }^{4}$, Geoffrey A. Kerchner², Monika Baudler², Paulo Fontoura ${ }^{2}$ and Rachelle Doody ${ }^{2,5}$

\begin{abstract}
Background: We previously investigated low doses (105 or $225 \mathrm{mg}$ ) of gantenerumab, a fully human monoclonal antibody that binds and removes aggregated amyloid- $\beta$ by Fc receptor-mediated phagocytosis, in the SCarlet RoAD (SR) and Marguerite RoAD (MR) phase 3 trials. Several lines of evidence suggested that higher doses may be necessary to achieve clinical efficacy. We therefore designed a positron emission tomography (PET) substudy to evaluate the effect of gantenerumab uptitrated to $1200 \mathrm{mg}$ every 4 weeks on amyloid- $\beta$ plaques as measured using florbetapir PET in patients with prodromal to moderate Alzheimer's disease (AD).

Methods: A subset of patients enrolled in the SR and MR studies who subsequently entered the open-label extensions (OLEs) were included in this substudy. Patients were aged 50 to 90 years with a clinical diagnosis of probable prodromal to moderate $A D$ and were included based on a visual read of the original screening scan in the double-blind phase. Patients were assigned to 1 of 5 titration schedules (ranging from 2 to 10 months) with a target gantenerumab dose of $1200 \mathrm{mg}$ every 4 weeks. The main endpoint of this substudy was change in amyloid$\beta$ plaque burden from OLE baseline to week 52 and week 104, assessed using florbetapir PET. Florbetapir global cortical signal was calculated using a prespecified standard uptake value ratio method converted to the Centiloid scale.
\end{abstract}

Results: Sixty-seven of the 89 patients initially enrolled had $\geq 1$ follow-up scan by August 15, 2018. Mean amyloid levels were reduced by 39 Centiloids by the first year and 59 Centiloids by year 2, a 3.5-times greater reduction than was seen after 2 years at $225 \mathrm{mg}$ in SR. At years 1 and 2,37\% and 51\% of patients, respectively, had amyloid- $\beta$ plaque levels below the amyloid- $\beta$ positivity threshold.

Conclusion: Results from this exploratory interim analysis of the PET substudy suggest that gantenerumab doses up to $1200 \mathrm{mg}$ resulted in robust amyloid- $\beta$ plaque removal at 2 years. PET amyloid levels were consistent with sparse-to-no neuritic amyloid- $\beta$ plaques in $51 \%$ of patients after 2 years of therapy. Amyloid reductions were similar to those observed in other placebo-controlled studies that have suggested potential clinical benefit.

Trial registration: ClinicalTrials.gov, NCT01224106 (SCarlet RoAD) and NCT02051608 (Marguerite RoAD).

Keywords: Alzheimer's disease, Amyloid- $\beta$ plaque, Centiloid, Disease-modification therapies, Gantenerumab, Florbetapir, Open-label extension, Positron emission tomography

\footnotetext{
* Correspondence: gregory.klein@roche.com

${ }^{1}$ Roche Pharma Research and Early Development, Basel, Switzerland

Full list of author information is available at the end of the article
}

(c) The Author(s). 2019 Open Access This article is distributed under the terms of the Creative Commons Attribution 4.0 International License (http://creativecommons.org/licenses/by/4.0/), which permits unrestricted use, distribution, and reproduction in any medium, provided you give appropriate credit to the original author(s) and the source, provide a link to the Creative Commons license, and indicate if changes were made. The Creative Commons Public Domain Dedication waiver (http://creativecommons.org/publicdomain/zero/1.0/) applies to the data made available in this article, unless otherwise stated. 


\section{Background}

Approximately 50 million people live with dementia worldwide, and this number is expected to grow to 82 million by 2030 and 152 million by 2050 [1]. Alzheimer's disease (AD) is the most common form of dementia, accounting for 60 to $70 \%$ of cases; together, $\mathrm{AD}$ and other types of dementia are the fifth leading cause of death worldwide [2]. Currently, there are no disease-modifying therapies that can delay disease course, prevent progression, or provide a cure [3]. Available pharmacological treatments for AD dementia offer mild symptomatic benefit with no effect on the underlying neuropathology of the disease [4-10].

AD pathophysiology is characterized by the progressive accumulation of amyloid- $\beta$ plaques predominantly comprising amyloid- $\beta$ peptides around neurons and the formation of intracellular neurofibrillary tangles containing pathologic tau protein [11]. Deposition of amyloid- $\beta$ plaques in the brain parenchyma is likely to occur decades before clinical symptoms manifest [11, 12]. The extent to which different amyloid- $\beta$ species contribute to the pathophysiology of AD remains uncertain [13], although in vitro and ex vivo evidence suggest both soluble oligomers and aggregated plaques are implicated in neurotoxic effects [13-15]. This hypothesis is being tested in multiple clinical trials evaluating drugs that can attenuate the accumulation and/or promote the removal of amyloid- $\beta$ in patients with AD [16-19].

Gantenerumab is a fully human anti-amyloid- $\beta$ IgG1 monoclonal antibody that binds with high affinity to aggregated amyloid- $\beta$ species and removes amyloid- $\beta$ plaques via $\mathrm{Fc} \gamma$ receptor-mediated microglial phagocytosis [20-22]. Gantenerumab neutralizes the neurotoxic effect of oligomeric amyloid- $\beta_{42}$ in vivo [22]. The effect of low-dose subcutaneous (SC) gantenerumab on cognition and function was investigated in 2 global phase 3 studies in patients with prodromal AD (SCarlet RoAD [SR]; NCT01224106; $n=799$ ) and mild AD (Marguerite RoAD [MR]; NCT02051608; $n=389$ ) [23, 24]. Dosing in SR was suspended after interim futility analyses revealed a low likelihood of meeting the primary endpoint at the doses studied (105 and $225 \mathrm{mg}$ SC every 4 weeks $[\mathrm{q} 4 \mathrm{w}])$. Recruitment for MR was subsequently stopped, although dosing continued. Overall, data gathered during the double-blind phase of SR suggest that doses studied were safe and well tolerated [23]. A dose-dependent decrease in brain amyloid- $\beta$ plaque burden was observed by week 100 (measured by positron emission tomography [PET]). Furthermore, dosedependent downstream pharmacodynamic effects were observed, including reductions in the cerebrospinal fluid biomarkers phosphorylated tau, total tau, and neurogranin [23]. A post hoc subgroup analysis of patients determined to be fast progressors in SR (according to Functional Assessment Questionnaire results, normalized hippocampal volume, and Clinical Dementia Rating Scale Sum of Boxes [CDR-SB] score at baseline [25]) also suggested a dose-dependent slowing of decline in the Alzheimer's Disease Assessment Scale cognitive subscale (ADAS-Cog) 13, Cambridge Neuropsychological Test Automated Battery, and MiniMental State Examination (MMSE) after 2 years of treatment [23, 25].

These observations suggest that higher doses of gantenerumab may have clinically relevant effects on cognition and function in patients with $\mathrm{AD}$, especially in the early stages of the disease [23]. Accordingly, open-label extension (OLE) phases incorporating PET substudies were initiated for SR and MR to assess the short- and long-term safety and pharmacodynamic effect of gantenerumab SC q4w uptitrated to a maximum dose of $1200 \mathrm{mg}$. Interim results of the ongoing, exploratory OLE PET substudies are reported here.

\section{Methods \\ Study design}

Complete study design and methodologic details of the MR and SR studies have been described elsewhere [23, 24]. Patients aged 50 to 90 years with a clinical diagnosis of probable prodromal or mild $\mathrm{AD}$ based on the $\mathrm{Na}$ tional Institute of Neurological and Communicative Disorders and Stroke/Alzheimer's Disease and Related Disorders Association criteria and a positive visual amyloid PET assessment from the original double-blind screening examinations of the MR and SR studies were eligible to enroll. Patients in SR who received doubleblind treatment and had $\geq 1$ follow-up visit and patients who were currently enrolled in MR were eligible for OLE participation. All patients entering the OLE at centers already involved in the SR and MR PET substudies of brain amyloid- $\beta$ imaging were eligible for the OLE PET substudy.

All SR and MR patients (including those previously on placebo) received subcutaneous gantenerumab in the OLE, which was uptitrated to a maximum dose of 1200 mg each month (Additional file 3: Figure S1). The dosetitration scheme used was based on patients' apolipoprotein $\mathrm{E}(A P O E)$ genotype (APOE\&4 carrier vs non-carrier) and the last double-blind treatment dose (gantenerumab $225 \mathrm{mg}$, gantenerumab $105 \mathrm{mg}$, or placebo). Gradual uptitration schemes were used to reach the target dose of $1200 \mathrm{mg}$ while decreasing the risk of adverse events, particularly amyloid-related imaging abnormalities (ARIA) representative of vasogenic edema (ARIA-E). The target dose was reached within 6 to 10 months in SR OLE patients and 2 to 6 months in MR OLE patients.

The work described was carried out in accordance with the Declaration of Helsinki. Written informed 
consent was provided by patients as deemed applicable by institutional review boards and/or independent ethics committees.

\section{Patient subgrouping and data cutoff}

At the start of the OLE studies, there were considerable differences between SR and MR patients with respect to prior exposure and stage of $\mathrm{AD}$. Therefore, for the purposes of this analysis, patients were divided into three groups. Patients in MR were divided into those who received active drug during the double-blind phase (MR double-blind active [MR-DBA]) and those who received placebo (MR double-blind placebo [MR-DBP]), while patients in SR were pooled together in a single group, regardless of assigned treatment in the double-blind phase, as they were all off treatment for 16 to 19 months prior to OLE higher dosing. The analyses presented here are based on a clinical data date of August 15, 2018.

\section{Safety and clinical treatment response monitoring}

Patients were monitored by one external independent monitoring committee until most patients reached the target dose, after which monitoring was continued by an internal monitoring committee. Disease severity and cognitive measures included the MMSE, CDR-SB, and ADAS-Cog 11. Safety was evaluated by adverse event reporting, blood safety testing, vital sign assessments, physical and neurological examinations, electrocardiography, and brain magnetic resonance imaging (MRI). Prior gantenerumab studies have shown that the most common safety-related events were ARIA and injectionsite erythema [23]. Interim safety results for the full SR and MR OLE populations have been reported separately and indicate that no new safety signals were identified compared with lower dosing levels [24, 26]. This work will report relations found between amyloid PET data and both ARIA and clinical treatment response in patients included in the PET substudy.

\section{Amyloid- $\beta$ plaque PET imaging}

Amyloid PET scans were scheduled at baseline (defined as prior to OLE day 1 dosing), week 52, and week 104 of OLE treatment. To minimize patient burden, a new OLE baseline PET scan was not required if the patient had received one during the double-blind phase within 9 to 12 months prior to OLE dosing. Therefore, for many patients, the duration between OLE baseline and week 52 is considerably longer than 52 weeks.

All PET data were obtained using a target dose of 370 $( \pm 10 \%)$ Becquerel $(\mathrm{MBq}){ }^{18} \mathrm{~F}$-florbetapir injected intravenously. A 15 -min scan was obtained starting at $50 \mathrm{~min}$ after ${ }^{18} \mathrm{~F}$-florbetapir injection. PET data were checked for quality and uniformly preprocessed; a global cortical signal was then calculated using a prespecified standard uptake value ratio (SUVR) method identical to the method reported for analysis in the SR double-blind study [21]. Briefly, that method computed the volumeweighted, gray matter-masked SUVR of six bilateral cortical regions from the Automated Anatomical Labelling (AAL) template, normalized by a cerebellar cortex reference region [27, 28]. The original screening 3D T1weighted MRI was segmented in the subject's space, then aligned to the AAL template space to perform the gray-matter masking.

To gain further insight and to allow better comparison of results from other studies, SUVR values were converted to Centiloid values. The Centiloid framework has the useful property of rescaling SUVR values obtained via different reference regions, methodologies, or even tracers to a common scale anchored by Centiloid values of 0 and 100 , which correspond to the transformed SUVR mean values of a young control group and AD group, respectively [29]. Briefly, paired florbetapir/PiB scans were downloaded from the GAAIN website (http://www.gaain.org/centiloidproject). SUVRs were calculated using the standard method from Klunk et al. [29] and the AAL template/gray matter-masked method used in this work. The Klunk SUVR method on level $1{ }^{11} \mathrm{C}$-Pib data (45 AD patients, 34 young control subjects) was replicated via a regression analysis with slope 0.994 and $R^{2}=0.99$. A subsequent regression analysis of the level 2 paired florbetapir $/{ }^{11} \mathrm{C}-\mathrm{PiB}$ allowed calculation of a single linear regression equation relating florbetapir AAL SUVR to Centiloid values. The linear transformations for this prespecified SUVR method are ${ }^{\mathrm{FBP}} \mathrm{SUVR}=0.514 \times{ }^{\mathrm{PiB}} \mathrm{SUVR}+0.749, R^{2}=0.75$; Centiloid $={ }^{\mathrm{FBP}}$ SUVR $\times 184.12-233.72[29,30]$, where ${ }^{\mathrm{FBP}}$ SUVR and ${ }^{\mathrm{PiB}} \mathrm{SUVR}$ represent the AAL-calculated SUVR for florbetapir, and ${ }^{\mathrm{PiB}}$ SUVR represents the Klunk SUVR method results for $\mathrm{PiB}$, respectively.

Another important anchor for interpreting PET results is the threshold for amyloid positivity, which is the quantitative threshold that best discriminates pathologically verified absence of plaques or sparse plaques from moderate to frequent plaques. Navitsky et al. showed that a Centiloid value of 24 corresponds to the amyloid positivity threshold for florbetapir [30]. Independently, the Alzheimer's Disease Neuroimaging Initiative (ADNI) PET core laboratory has established a positivity threshold of 1.11 by using a FreeSurfer method, whole cerebellum reference, and a corresponding Centiloid value of 24 [31]. Using the florbetapir data available on the GAAIN website (13 young controls, 33 elderly subjects) and calculating SUVR using both the ADNI FreeSurfer and AAL SUVR processing pipelines, it is found that the 1.11 ADNI positivity threshold corresponds to a 1.40 value using the AAL method with a cerebellar cortex reference (Additional file 4: Figure S2) [32]. Transformed into Centiloids, the 1.40 SUVR also corresponds to a 24Centiloid threshold. 


\section{Statistical analysis}

The analysis population included all study participants receiving $\geq 1$ OLE follow-up scan. All analyses presented here are exploratory in nature. Descriptive statistics were used to characterize patient demographics as well as change from baseline in PET Centiloid value and CDR-SB, ADAS-Cog 11, and MMSE scores. PET Centiloid value was also analyzed using a mixed model for repeated measures (MMRM), with visit, treatment group, and the interaction for treatment group by visit as independent variables. An unstructured covariance matrix was used to capture withinpatient correlation. For descriptive group comparisons, the MMRM model was also applied to change from baseline in the clinical endpoint, with baseline value, group, and group-by-visit interaction as independent variables. Joint linear mixed effects (JLME) models were used to evaluate the association between the annual rate of change in PET and that in CDRSB, ADAS-Cog 11, and MMSE scores while simultaneously controlling the impact of the PET baseline on the rate of change in the latter. For the ease of model convergence, PET, CDR-SB, ADAS-Cog 11, and MMSE scores were standardized to $z$-scores using the baseline mean and SD.

\section{Results}

\section{Patients}

A total of 379 patients were dosed in the SR and MR OLE trials; of these, 67 had scans at OLE week 52 and 39 had scans at OLE week 104 as of August 15, 2018 (Additional file 3: Figure S1). Patient characteristics at OLE baseline are summarized for OLE week 52 completers in Table 1. Amyloid loads at OLE baseline were 91, 80, and 50 Centiloids in the MRDBP, MR-DBA, and SR groups, respectively (Table 1).
Mean (SD) MMSE scores were 21.6 (4.5), 19.3 (5.0), and 18.8 (4.8), respectively, and ranged from 11 to 29. This indicates that although the original patient populations in the double-blind studies were prodromal/mild, some patients' health had declined by the time of OLE baseline, resulting in an overall mild/moderate patient population for this analysis. All OLE baseline characteristics of week 52 completers were broadly consistent with those of week 104 completers, except for mean baseline Centiloid value in the SR cohort, which was 70 in week 104 completers (not shown) but only 50 in week 52 completers.

\section{Gantenerumab markedly reduced brain amyloid- $\beta$ plaque levels}

The reduction in amyloid PET was significant in all subgroups and at all time points. Mean (SE) PET Centiloid reductions from baseline were -42 (7.1), -48 (8.0), and -21 (8.3) at week 52 and - 71 (7.7), - 61 (8.9), and $-34(8.9)$ at week 104 in the MR-DBP, MR-DBA, and SR groups, respectively (Fig. 1); consistent results were observed for week 104 completers (Additional file 1: Table S1). Example images from five patients are shown in Fig. 2. Individually, all week 104 completers had reduced amyloid, with reductions ranging from 11 to 169 Centiloids, and 51\% of patients had amyloid levels below the positivity threshold at week 104 (Fig. 1b). A strong correlation was seen between higher amyloid load at OLE baseline and greater amyloid reduction during the first year of gantenerumab treatment (Additional file 5: Figure S3). Large regional reductions were seen throughout the brain regions primarily involved in $\mathrm{AD}$, with the greatest reductions observed in the anterior cingulate (Additional file 6: Figure S4). There were no observed effects of APOE status on PET reduction.

Table 1 OLE baseline characteristics of patients with PET scan at OLE week 52

\begin{tabular}{|c|c|c|c|}
\hline Characteristic & MR-DBP $(n=27)$ & $\operatorname{MR}-\mathrm{DBA}(n=21)$ & $\mathrm{SR}(n=19)$ \\
\hline Age at start of OLE, median $(\mathrm{IQR})$, years & $74(69-77)$ & $65(59-79)$ & $72(70-78)$ \\
\hline Female, $n(\%)$ & $12(44)$ & $11(52)$ & $11(58)$ \\
\hline White, $n(\%)$ & $27(100)$ & $20(95)$ & $19(100)$ \\
\hline \multicolumn{4}{|l|}{ APOE\&4 genotype, $n(\%)$} \\
\hline 0 & $9(33)$ & $10(48)$ & $2(11)$ \\
\hline 1 & $13(48)$ & $4(19)$ & $14(74)$ \\
\hline 2 & $5(19)$ & $7(33)$ & $3(16)$ \\
\hline MMSE score at OLE baseline, mean (SD) & $21.6(4.5)$ & $19.3(5.0)$ & $18.8(4.8)$ \\
\hline Centiloid at OLE baseline, mean (SD) & $91.1(46.8)$ & $79.6(51.6)$ & $49.6(52.8)$ \\
\hline Duration on high dose, median (IQR), weeks ${ }^{a}$ & $35(33-37)$ & $42(40-45)$ & $25(21-33)$ \\
\hline Duration between baseline and week 52 PET scan, median (IQR), weeks & $68(60-82)$ & $73(64-88)$ & $55(54-58)$ \\
\hline Patients with baseline PET scan below amyloid- $\beta$ threshold, $n$ (\%) & 0 & $3(14)$ & $7(37)$ \\
\hline
\end{tabular}

${ }^{a}$ High dose: $\geq 6$ doses ( 6 months) of 900 to $1200 \mathrm{mg}$ gantenerumab 


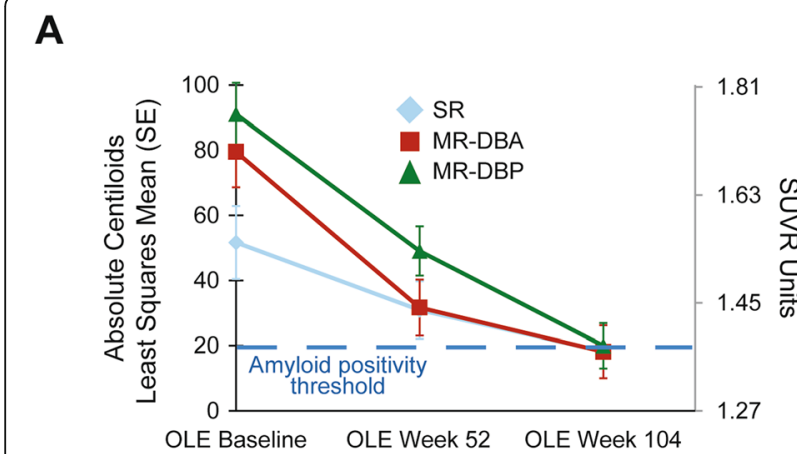

Absolute Centiloids, Least Squares Mean (SE)

\begin{tabular}{lccc}
\hline SR & $51.7(11.2)$ & $\begin{array}{c}30.9(8.9) \\
n=19 \\
n=0.0148\end{array}$ & $\begin{array}{c}18.2(8.1) \\
n=12 \\
p=0.004 \\
\end{array}$ \\
& & vs. OLE baseline & vs. OLE baseline \\
MR-DBA & $79.6(10.9)$ & $31.7(8.6)$ & $18.1(8.1)$ \\
& $n=21$ & $n=21$ & $n=11$ \\
& & $p<0.0001$ & $p<0.0001$ \\
& & vs. OLE baseline & vs. OLE baseline \\
MR-DBP & $91.1(9.6)$ & $49.1(7.6)$ & $19.9(7.0)$ \\
& $n=27$ & $n=27$ & $n=16$ \\
& & $p<0.0001$ & $p<0.0001$ \\
& & vs. OLE baseline & vs. OLE baseline
\end{tabular}

B

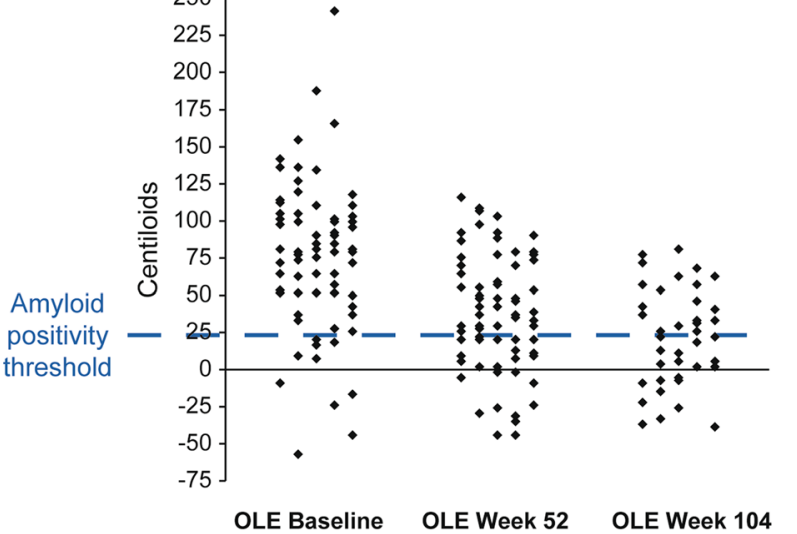

$\begin{array}{rccc}\text { No. of patients } & 67 & 67 & 39 \\ \text { SR } & 19 & 19 & 12 \\ \text { MR-DBA } & 21 & 21 & 11 \\ \text { MR-DBP } & 27 & 27 & 16 \\ \text { below threshold } & 15 \% & 37 \% & 51 \% \\ \text { SR } & 37 \% & 58 \% & 50 \% \\ \text { MR-DBA } & 14 \% & 33 \% & 55 \% \\ \text { MR-DBP } & 0 \% & 26 \% & 50 \%\end{array}$

Fig. 1 Marked and consistent reduction of amyloid load in patients receiving high-dose gantenerumab. a Marked reduction of amyloid- $\beta$ plaques in patients receiving high-dose gantenerumab, and consistent reduction of amyloid- $\beta$ plaques in all patient groups. $\mathbf{b}$ Reduction of amyloid- $\beta$ plaque burden to below positivity threshold following high-dose gantenerumab. ${ }^{a}$ Analyzed using a mixed model for repeated measures

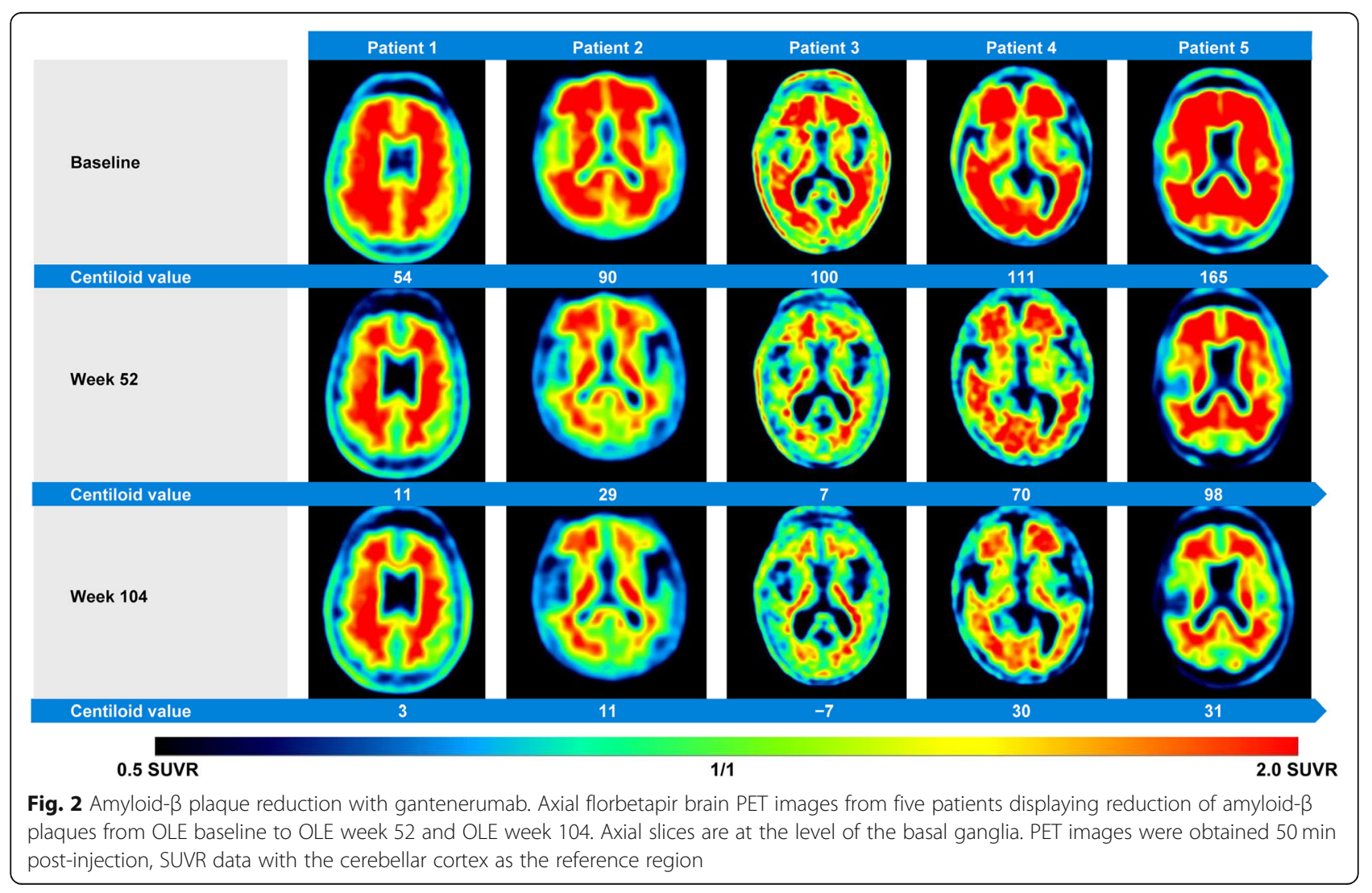




\section{Amyloid reduction and ARIA}

In our analysis population of OLE PET week 52 substudy completers, 28 patients experienced ARIA-E, including 7 who reported symptoms. Global amyloid load at OLE baseline was not significantly different between the ARIA-E and non-ARIA-E groups (Table 2), and there were no significant differences between the ARIA-E and non-ARIA-E groups at either week 52 or 104; however, a directional trend toward slightly higher reductions in the ARIA-E group was observed (Table 2).

\section{Amyloid reduction and clinical results}

This open-label, non-placebo-controlled trial was not designed to support analyses of clinical efficacy. However, exploratory analyses of clinical endpoints were performed. Baseline and follow-up CDR-SB, ADASCog 11, and MMSE values are summarized in Table 3. Correlation analysis of change from baseline to week 104 among completers (patients with non-missing OLE week 104 PET data) suggested a directional trend for slower clinical decline with higher amyloid removal for all three endpoints (Fig. 3a, d, g).

"Higher" and "lower" PET amyloid reduction groups were defined based on the median PET Centiloid change from baseline to week 52 (Fig. 3c, f, i; median change threshold, -34.99 Centiloids) or week 104 (Fig. 3b, e, h; median change threshold, - 55.23 Centiloids), among completers. The "higher" versus "lower" dichotomization was performed at two different time points to test the hypothesis that clinical benefit may follow amyloid reduction temporally [33]; in other words, higher amyloid reduction at week 52 may be associated with greater slowing of clinical decline at week 104, whereas this effect may be less pronounced when the time point for dichotomization of amyloid reduction and the clinical evaluation are contemporaneous. At week 104, point estimates for CDR-SB, ADAS-Cog 11, and MMSE showed numerically less decline in the higher vs lower PET amyloid reduction groups, defined at either OLE week 52 or 104. The differences between the higher and lower PET groups were non-significant with respect to change from baseline on all three clinical endpoints. When pooled together, results from the JLME analysis indicated a directional but not significant association between the annual reduction in $\mathrm{PET}$ and the annual change in CDR-SB, ADAS-Cog 11, and MMSE. For instance, an annual reduction of $0.54 \mathrm{SD}$ in standardized $z$-score PET Centiloid would lead to a slowing of $0.55 \mathrm{SD}$ in the standardized $z$-score ADAS-Cog increase. However, with the limited sample size and absence of a placebo arm, robust interpretation and conclusion from any such analysis is not possible.

\section{Discussion}

This PET substudy of the MR and SR OLE studies investigated the effect of higher-dose gantenerumab on amyloid- $\beta$ plaques over 104 weeks in patients with prodromal to moderate AD dementia. Reductions in PET values to below the amyloid- $\beta$ positivity threshold were achieved in $37 \%$ of patients at week 52 and $51 \%$ at week 104 , suggesting that amyloid- $\beta$ plaques can be reduced to levels that would not support a neuropathological diagnosis of $\mathrm{AD}$ [34]. In addition, amyloid reductions were consistently observed across most patients, indicating that gantenerumab has the potential to reduce amyloid- $\beta$ plaques as suggested by its proposed mechanism of action of preferential binding to amyloid- $\beta$ aggregates and consequent plaque removal.

Compared with the PET mean (SD) change observed with the 225-mg dose during the SR 2-year double-blind period (-0.09 [0.14] SUVR, - 16.6 [25.8] Centiloids) [23], reductions in this OLE substudy were markedly higher, with an overall reduction in all three cohorts at week 104 of 59.0 (35.3) Centiloids. The 64.8\%, 77.3\%, and $78.2 \%$ amyloid reductions in the SR, MR-P, and MR-NP groups, respectively, were well beyond the $2.4 \% \pm 1.41 \%$ test-retest data reported for florbetapir [35]. Since patients in all three OLE cohorts achieved reductions in amyloid load at week 104 to near or below the positivity threshold, the lower mean reductions in the MR-DBA and SR groups compared with the MRDBP group were most likely due to their lower baseline amyloid loads and not due to a difference in treatment effect among the three groups. The lower comparative reduction in the SR group at week 52 could also be explained by the shorter time on high dose compared with the MR-DBA and MR-DBP groups. Regional analyses indicate that amyloid reductions are fairly uniform throughout the regions of the brain known to have amyloid, indicating that sufficient blood-brain barrier penetration of gantenerumab has occurred to effect globally large amyloid reductions. Regarding PET amyloid reductions and ARIA-E, it was observed that while patients with ARIA-E may have a trend toward slightly higher amyloid reductions compared with patients without ARIA-E, both groups achieved very large amyloid reductions. Consequently, the gantenerumab data indicate that ARIA-E is not a prerequisite for large amyloid reductions. This result contrasts the data reported for bapineuzumab, in which there was a lack of change from baseline in the non-ARIA-E groups [36].

Although comparison of PET amyloid reductions with gantenerumab vs the effect seen with other anti-amyloid therapies is challenging [37], use of the Centiloid scale helps to better understand the current landscape of therapies with amyloid removal as a mechanism of action $[29,38]$. To date, three other compounds have 


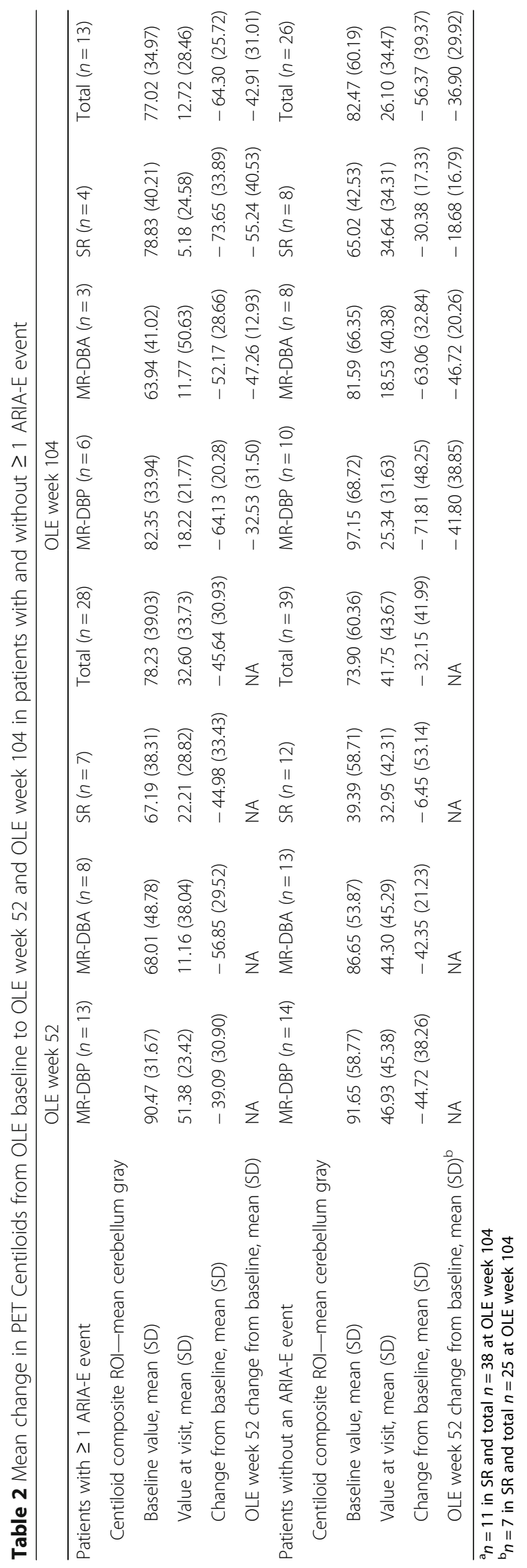




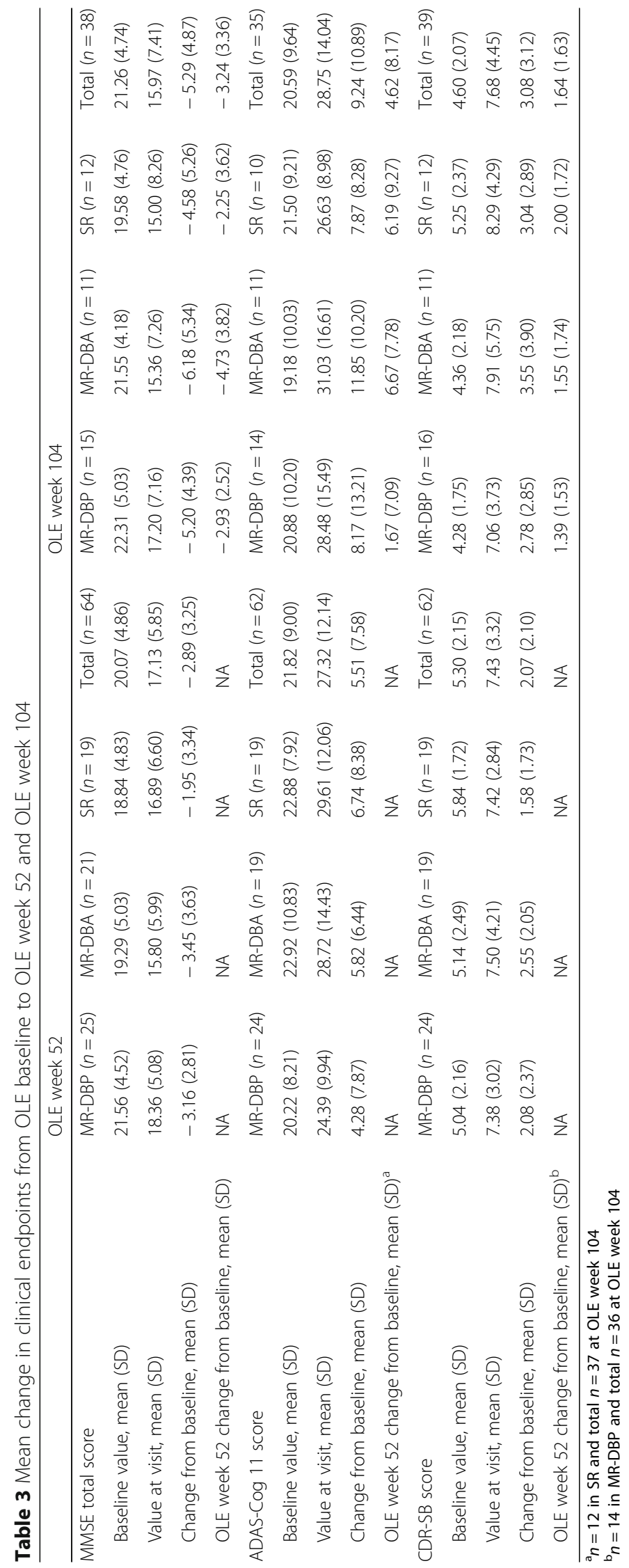



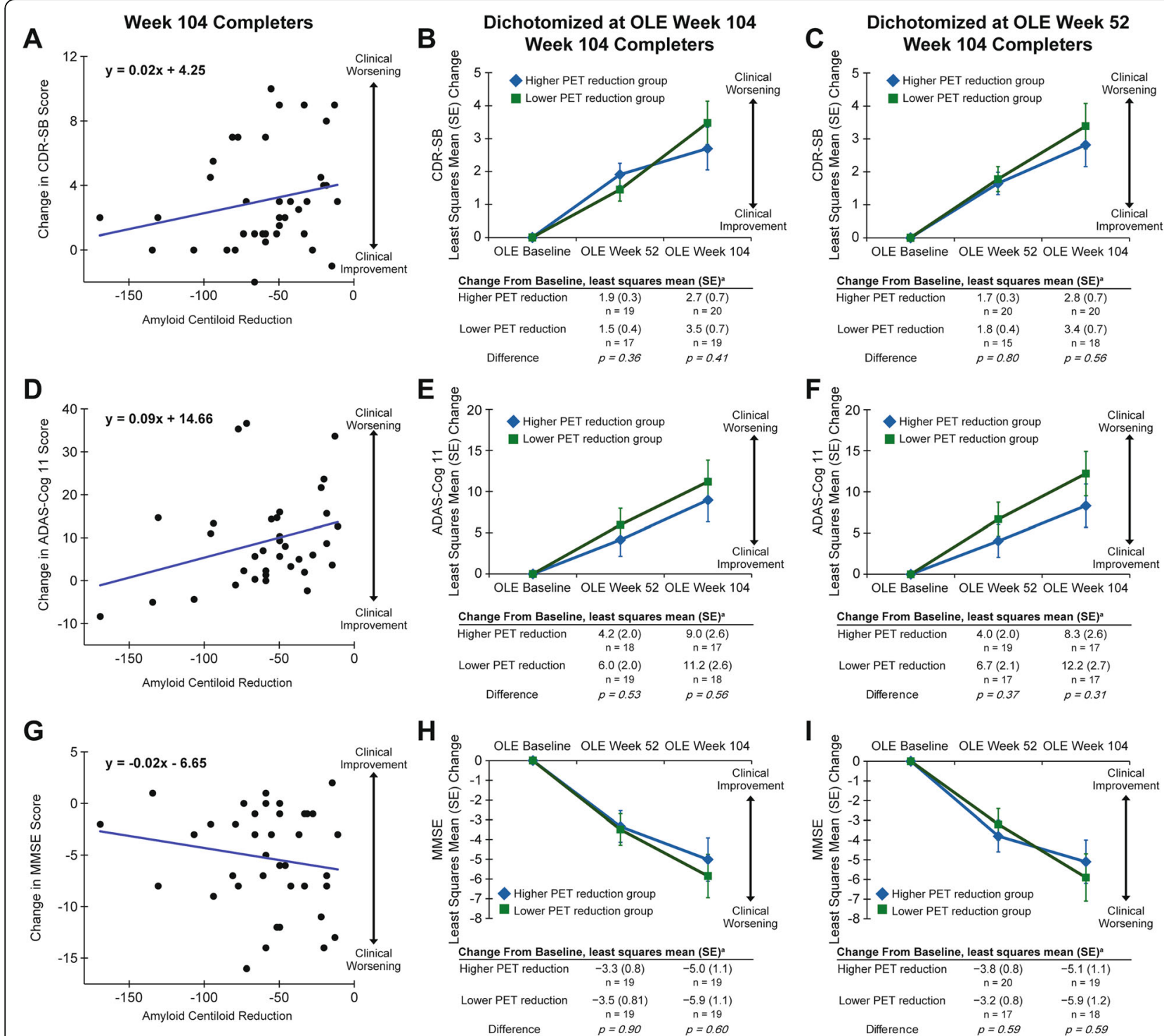

Fig. 3 Association of PET and clinical results. a, d, g Regression analysis of CDR-SB, ADAS-Cog 11, and MMSE, respectively, shows directional slowing of clinical decline with increasing amyloid reduction. b, e, h Change in CDR-SB, ADAS-Cog 11, and MMSE for week 104 completers stratified by patients with high and low amyloid reduction at week 104 also shows directional slowing of clinical decline in the group with higher amyloid reduction. $\mathbf{c}, \mathbf{f}, \mathbf{i}$ Stratification of patients by amyloid reduction at the earlier week 52 time point shows increased clinical benefit in the high PET reduction group, suggesting a temporal lag between amyloid reduction and clinical benefit

reported similar levels of amyloid reduction. The 10-mg/ $\mathrm{kg}$ arm of the aducanumab PRIME phase $1 \mathrm{~b}$ study had a baseline Centiloid value of 96 (SUVR, 1.44) and a reduction of 71 Centiloids at week $110[33,39]$. The highestdose group for BAN2401 had a 70-Centiloid reduction at 18 months from 74.5 Centiloids at baseline [40], while the LY3002813 highest-dose group showed a 68.8-Centiloid reduction from 111 Centiloids at baseline by week 24 [41]. The most representative gantenerumab OLE cohort for comparison is the previously untreated MR-DBP arm, which had a baseline load of 92 Centiloids and a reduction of 69 Centiloids by week 104 . When comparing Centiloid reductions seen here to those in other studies, it is important to note that the rate of reduction appears to be related to baseline levels of amyloid as well as to drug effects.

Earlier anti-amyloid- $\beta$ studies, including bapineuzumab and solanezumab, as well as BACE-1 studies, such as verubecestat, did not report amyloid values using Centiloid values, but all three of these showed no or modest amyloid$\beta$ reductions, $<5 \%$ with respect to baseline after treatment [42-44]. Other studies have reported amyloid decline in placebo groups [45], but the magnitude was far smaller than the amyloid reductions seen with the four high amyloid-removing monoclonal antibodies listed above. 
There are several limitations to these analyses. The OLE studies reported here did not have any disease severity-related inclusion or exclusion criteria, and by the start of the OLE, some patients had progressed to a mild-moderate stage. The studies were neither randomized nor blinded and did not include a placebo arm; furthermore, the studies are still ongoing, with additional subjects expected to reach week 104. In addition, several measures could be considered to improve the robustness of the results. Results reported here are from static PET imaging, which may not account for certain drug effects (e.g., changes in blood flow or clearance). Dynamic imaging may produce slightly more robust results; however, the differences due to perfusion changes are likely to be small compared with the SUVR change seen here [4648]. Our use of static PET is consistent with all previously reported findings on amyloid reduction with investigational AD therapies [33, 40, 43, 49, 50]. Brain atrophy progresses over time in $\mathrm{AD}$, potentially even after amyloid aggregates are removed, and application of a partial volume correction (PVC) may be useful for interpreting our findings. PVC will be considered for future exploratory analysis. It should be noted, however, that evidence supporting PVC is mixed, with some reporting an improved signal [51] whereas others report greater noise [52, 53]. Furthermore, an analysis of cerebellar gray, pons, and white matter reference region SUVs compared to cortical target region SUVs (see Additional file 2: Table S2) shows that the SUVR change is primarily driven by reductions of signal in the target cortical regions, not the reference regions, and is not likely driven by spillover of cortical white matter activity.

A key step is to clarify how amyloid reductions, such as those reported here, correspond to any clinical benefit among patients with early symptomatic AD. Other randomized, parallel-group studies with aducanumab [39] and BAN2401 [40] have demonstrated PET amyloid signal reduction accompanied by indications of clinical efficacy in a similar patient population. In addition, a post hoc analysis of the SR trial demonstrated evidence of gantenerumab dose-dependent slowing of decline in some clinical efficacy scales [23]. Whereas two phase III studies of aducanumab were discontinued due to a low likelihood of reaching the primary endpoint [54], initially calling into question the relationship of amyloid reduction to clinical benefit, a subsequent analysis of the same studies reported evidence of an exposure-dependent clinical benefit associated with amyloid reduction [55]. (Full data from these phase III studies have not yet been published at the time of this writing.)

In this study, exploratory results showed a directional trend, whereby patients with higher PET amyloid reduction exhibited numerically less cognitive decline. Considering the limited sample size, heterogeneity among patients, and other potential confounders, including the observed relationship between baseline amyloid levels and amyloid reduction, caution must be used in the interpretation of clinical outcome data, particularly the comparison to previously published results. Nevertheless, these data do contribute to an overall body of evidence in the field that amyloid reduction is associated with clinical benefit among patients with early symptomatic $\mathrm{AD}$, as outlined above. The hypothesis that higherdose gantenerumab treatment contributes to clinical benefit in association with amyloid reduction is currently being tested in the ongoing GRADUATE phase III pivotal studies (NCT03444870, NCT03443973).

\section{Conclusions}

We have shown that gantenerumab reduces amyloid- $\beta$ plaques, one of the main pathological hallmarks of AD. These data and the favorable safety profile observed at these dose levels provide the rationale for further investigation of the clinical efficacy of gantenerumab, and two pivotal phase III trials in patients with early AD (GRADUATE 1 and 2) are currently ongoing [56, 57].

\section{Supplementary information}

Supplementary information accompanies this paper at https://doi.org/10. 1186/s13195-019-0559-z.

\section{Additional file 1: Table S1. Reduction in Amyloid Load in Patients Receiving High-Dose Gantenerumab (Week 104 Completers). Findings in Week 104 completers show continued reduction in amyloid load across groups over 104 weeks, consistent with the overall group.}

Additional file 2: Table S2. SUV Values for Week 104 Completers. Evaluation of cerebellar gray, pons, and white matter reference region SUVs compared with cortical target region SUVS show that SUVR change is primarily driven by changes in the target cortical regions.

Additional file 3: Figure S1. Study Design With Dosing Schedule and Patient Disposition. (A) Schematic representation of the MR and SR OLE study designs and dose-titration schedules. All patients in the OLE (including those previously on placebo) received gantenerumab subcutaneously every 4 weeks. Dose-titration schedules for uptitration to $1200 \mathrm{mg}$ were assigned based on APOE\&4 carrier status and last treatment dose during the doubleblind phase. (B) Patient disposition. ${ }^{a}$ Including 1 patient who missed their week 52 visit.

Additional file 4: Figure S2. Linear Regression of AAL SUVR (cerebellar gray reference) vs ADNI FreeSurfer (whole cerebellum reference). Linear regression of SUVR results computed on the same ADNI patients allows transformation of the previously published 1.11 amyloid- $\beta$ positivity threshold to a value of 1.40 for the method here using a cerebellar cortex reference region. The $95 \% \mathrm{Cls}$ (shaded area) were calculated using the bootstrap (quartile) method.

Additional file 5: Figure S3. Correlation Between Amyloid Load at OLE Baseline and Amyloid Change Over Time. Rate of amyloid reduction during the first year of gantenerumab treatment appears to be linked to baseline amyloid burden. Higher rates of amyloid reduction are seen with greater baseline burden.

Additional file 6: Figure S4. Regional Reductions in Amyloid Load. Amyloid reductions are seen in all regions known to be involved with amyloid pathology. Highest reductions are seen in the cingulate, frontal, and striatum areas. When adjusted for baseline amyloid burden, the caudate region shows the greatest regional reduction. 


\section{Abbreviations}

AAL: Automated Anatomical Labelling; AD: Alzheimer's disease; ADASCog: Alzheimer's Disease Assessment Scale cognitive subscale; ADNI: Alzheimer's Disease Neuroimaging Initiative; APOE\&4: Apolipoprotein E ع4; ARIA-E: Amyloid-related imaging abnormalities representative of vasogenic edema; CDR-SB: Clinical Dementia Rating Scale Sum of Boxes; LONI: Laboratory of Neuroimaging; MMSE: Mini-Mental State Examination; MR: Marguerite RoAD; MR-DBA: Marguerite RoAD double-blind active arm; MR-DBP: Marguerite RoAD double-blind placebo arm; NA: Not applicable; OLE: Open-label extension; PET: Positron emission tomography; q4w: Every 4 weeks; ROI: Region of interest; SC: Subcutaneous; SE: Standard error; SR: SCarlet RoAD; SUVR: Standard uptake value ratio

\section{Acknowledgements}

The authors wish to thank the patients, caregivers, and investigators in the SCarlet RoAD and Marguerite RoAD open-label extension studies. The authors acknowledge the contributions of John Seibyl and Ken Marek of Invicro, Boston, MA. Medical writing support was provided by Jonathan Plumb, PhD, of Bioscript Medical, Macclesfield, UK, and Liz LaFlamme, PhD, of Health Interactions, Hamilton, NJ, and was funded by Roche Pharmaceuticals.

\section{Authors' contributions}

All authors meet the ICMJE criteria and were involved in either the conception or the design of the study, writing the study report, data collection, or data analysis and interpretation. All authors critically reviewed and edited the manuscript and approved the final version for submission.

\section{Funding}

The SCarlet RoAD and Marguerite RoAD studies were funded by F. Hoffmann-La Roche Ltd.

\section{Availability of data and materials}

The datasets analyzed during the current study are available from the corresponding author on reasonable request. Qualified researchers may request access to individual patient level data through the clinical study data request platform (www.clinicalstudydatarequest.com). Further details on Roche's criteria for eligible studies are available here (https:// clinicalstudydatarequest.com/Study-Sponsors/Study-Sponsors-Roche.aspx). For further details on Roche's Global Policy on the Sharing of Clinical Information and how to request access to related clinical study documents, see here (https://www.roche.com/research_and_development/who_we_are_ how_we_work/clinical_trials/our_commitment_to_data_sharing.htm).

\section{Ethics approval and consent to participate}

The study protocol was approved by the respective institutional review boards prior to participant recruitment and was conducted in accordance with US Food and Drug Administration regulations, International Council on Harmonization E6 Guideline for Good Clinical Practice, and applicable local, state, federal, and country laws.

\section{Consent for publication}

Not applicable

\section{Competing interests}

GK, PD, SRi, CH, DA-S, MA, MB, and PF are full-time employees of F. Hoffmann-La Roche Ltd. GK, CH, DA-S are shareholders in F. Hoffmann-La Roche Ltd. SRe and NV are full-time employees of Roche Products Ltd. RD is a full-time employee and shareholder in F. Hoffmann-La Roche Ltd. and Genentech Inc. GW is the statistician for the DIAN-TU study and has no competing interests to disclose. RB is a principal investigator of a therapeutic trial evaluating investigational drugs. Washington University is also the regulatory sponsor for this trial. Funding for DIAN-TU clinical trials includes the National Institute on Aging of the National Institutes of Health, Alzheimer's Association, Eli Lilly and Co, F. Hoffmann-La Roche Ltd. and Genentech, Inc., Janssen, Avid Radiopharmaceuticals, GHR Foundation, and an anonymous foundation. Washington University and Dr. David Holtzman, Department Head of Neurology at Washington University School of Medicine, may receive royalty income for the investigational drug, solanezumab, which is being tested in the DIAN-TU trial and was developed by Dr. Holtzman and licensed by Washington University to Eli Lilly \& Company. RB also receives research funding from the DIAN-TU Pharma Consortium (AbbVie, Biogen, Eisai, Eli Lilly and Co/Avid Radiopharmaceuticals, F. Hoffmann-La Roche Ltd. and Genentech, Inc.,
Janssen, and United Neuroscience); and has received honoraria from Janssen, Pfizer, and F. Hoffmann-La Roche Ltd. as a speaker, Eisai as a consultant, and from Merck, F. Hoffmann-La Roche Ltd., and Pfizer as an Advisory Board member.

\section{Author details}

${ }^{1}$ Roche Pharma Research and Early Development, Basel, Switzerland. ${ }^{2}$ Roche/ Genentech Product Development, Neuroscience, Basel, Switzerland. ${ }^{3}$ Roche Products Ltd, Welwyn Garden City, UK. "Washington University School of Medicine in St. Louis, St. Louis, MO, USA. ${ }^{5}$ Genentech, Inc., South San Francisco, CA, USA.

Received: 24 July 2019 Accepted: 19 November 2019 Published online: 12 December 2019

\section{References}

1. World Health Organization (WHO). Dementia fact sheet. 19 September 2019. https://www.who.int/news-room/fact-sheets/detail/dementia. Accessed 31 Oct 2019.

2. World Health Organization (WHO). The top 10 causes of death. 24 May 2018. http://www.who.int/en/news-room/fact-sheets/detail/the-top-10causes-of-death. Accessed 31 Oct 2019.

3. GBD 2015 Mortality and Causes of Death Collaborators. Global, regional, and national life expectancy, all-cause mortality, and cause-specific mortality for 249 causes of death, 1980-2015: a systematic analysis for the Global Burden of Disease Study 2015. Lancet. 2016;388:1459-544.

4. Birks JS, Melzer D, Beppu H. Donepezil for mild and moderate Alzheimer's disease. Cochrane Database Syst Rev. 2000;4:CD001190.

5. Birks J. Cholinesterase inhibitors for Alzheimer's disease. Cochrane Database Syst Rev. 2006;1:CD005593.

6. Loy C, Schneider L. Galantamine for Alzheimer's disease and mild cognitive impairment. Cochrane Database Syst Rev. 2006;1:CD001747.

7. Kurz A, Perneczky R. Amyloid clearance as a treatment target against Alzheimer's disease. J Alzheimers Dis. 2011;24(Suppl 2):61-73.

8. Neugroschl J, Sano M. Current treatment and recent clinical research in Alzheimer's disease. Mt Sinai J Med. 2010;77:3-16.

9. Corbett A, Smith J, Ballard C. New and emerging treatments for Alzheimer's disease. Expert Rev Neurother. 2012;12:535-43.

10. Francis PT, Palmer AM, Snape M, Wilcock GK. The cholinergic hypothesis of Alzheimer's disease: a review of progress. J Neurol Neurosurg Psychiatry. 1999;66:137-47.

11. Bateman RJ, Xiong C, Benzinger TL, Fagan AM, Goate A, Fox NC, et al. Clinical and biomarker changes in dominantly inherited Alzheimer's disease. N Engl J Med. 2012;367:795-804.

12. Villemagne $V L$, Burnham $S$, Bourgeat $P$, Brown B, Ellis KA, Salvado O, et al. Amyloid beta deposition, neurodegeneration, and cognitive decline in sporadic Alzheimer's disease: a prospective cohort study. Lancet Neurol. 2013;12:357-67.

13. Haass C, Selkoe DJ. Soluble protein oligomers in neurodegeneration: lessons from the Alzheimer's amyloid beta-peptide. Nat Rev Mol Cell Biol. 2007;8:101-12.

14. Benilova I, Karran E, De Strooper B. The toxic A oligomer and Alzheimer's disease: an emperor in need of clothes. Nat Neurosci. 2012;15:349-57.

15. Wang S, Mims PN, Roman RJ, Fan F. Is beta-amyloid accumulation a cause or consequence of Alzheimer's disease? J Alzheimers Parkinsonism Dement. 2016;1:007.

16. Selkoe DJ, Hardy J. The amyloid hypothesis of Alzheimer's disease at 25 years. EMBO Mol Med. 2016;8:595-608.

17. Kopeikina KJ, Hyman BT, Spires-Jones TL. Soluble forms of tau are toxic in Alzheimer's disease. Transl Neurosci. 2012;3:223-33.

18. Sevigny J, Suhy J, Chiao P, Chen T, Klein G, Purcell D, et al. Amyloid PET screening for enrichment of early-stage Alzheimer disease clinical trials: experience in a phase 1b clinical trial. Alzheimer Dis Assoc Disord. 2016;30:1-7.

19. Cummings $J$, Cohen S, Van Dyck CH, Brody M, Curtis C, Cho W, et al. ABBY: a phase 2 randomized trial of crenezumab in mild to moderate Alzheimer disease. Neurology. 2018;90:e1889-e97.

20. Novakovic D, Feligioni M, Scaccianoce S, Caruso A, Piccinin S, Schepisi C, et al. Profile of gantenerumab and its potential in the treatment of Alzheimer's disease. Drug Des Devel Ther. 2013;7:1359-64.

21. Ostrowitzki S, Deptula D, Thurfjell L, Barkhof F, Bohrmann B, Brooks DJ, et al. Mechanism of amyloid removal in patients with Alzheimer disease treated with gantenerumab. Arch Neurol. 2012;69:198-207. 
22. Bohrmann B, Baumann K, Benz J, Gerber F, Huber W, Knoflach F, et al. Gantenerumab: a novel human anti-A $\beta$ antibody demonstrates sustained cerebral amyloid- $\beta$ binding and elicits cell-mediated removal of human amyloid- $\beta$. J Alzheimers Dis. 2012;28:49-69.

23. Ostrowitzki S, Lasser RA, Dorflinger E, Scheltens P, Barkhof F, Nikolcheva T, et al. A phase III randomized trial of gantenerumab in prodromal Alzheimer's disease. Alzheimers Res Ther. 2017;9:95.

24. Abi-Saab D, Andjelkovic M, Pross N, Delmar P, Voyle N, Esau N, et al. MRI findings in the open label extension of the Marguerite RoAD study in patients with mild Alzheimer's disease. J Prev Alz Dis. 2017;4:339 (P36).

25. Delor I, Charoin JE, Gieschke R, Retout S, Jacqmin P. Modeling Alzheimer's disease progression using disease onset time and disease trajectory concepts applied to CDR-SOB scores from ADNI. CPT Pharmacometrics Syst Pharmacol. 2013;2:e78.

26. Andjelkovic M, Abi-Saab D, Pross N, Delmar P, Voyle N, Mertes M, et al. Safety and tolerability of gantenerumab in an open-label extension of SCarlet RoAD trial, a global study in patients with prodromal disease. J Prev Alz Dis. 2017;4:301 (OC26).

27. Fleisher AS, Chen K, Liu X, Roontiva A, Thiyyagura P, Ayutyanont N, et al. Using positron emission tomography and florbetapir F18 to image cortical amyloid in patients with mild cognitive impairment or dementia due to Alzheimer disease. Arch Neurol. 2011;68:1404-11.

28. Barthel H, Gertz HJ, Dresel S, Peters O, Bartenstein P, Buerger K, et al. Cerebral amyloid- $\beta$ PET with florbetaben (18F) in patients with Alzheimer's disease and healthy controls: a multicentre phase 2 diagnostic study. Lancet Neurol. 2011;10:424-35.

29. Klunk WE, Koeppe RA, Price JC, Benzinger TL, Md D Sr, Jagust WJ, et al. The Centiloid Project: standardizing quantitative amyloid plaque estimation by PET. Alzheimers Dement, 2015. 11:1-15 e1-4.

30. Navitsky M, Joshi AD, Kennedy I, Klunk WE, Rowe CC, Wong DF, et al. Standardization of amyloid quantitation with florbetapir standardized uptake value ratios to the Centiloid scale. Alzheimers Dement. 2018;14: $1565-71$.

31. Landau S, Jagust W. Alzheimer's Disease Neuroimaging Initiative (ADNI). Florbetapir processing methods. Rev June 25 2015. https://adni.bitbucket.io/ reference/docs/UCBERKELEYAV45/ADNI_AV45_Methods_JagustLab_06.25.15. pdf. Accessed 31 Oct 2019.

32. Clark CM, Schneider JA, Bedell BJ, Beach TG, Bilker WB, Mintun MA, et al. Use of florbetapir-PET for imaging beta-amyloid pathology. JAMA. 2011;305: 275-83.

33. Sevigny J, Chiao P, Bussière T, Weinreb PH, Williams L, Maier M, et al. The antibody aducanumab reduces $A \beta$ plaques in Alzheimer's disease. Nature. 2016;537:50-6.

34. Clark CM, Pontecorvo MJ, Beach TG, Bedell BJ, Coleman RE, Doraiswamy PM, et al. Cerebral PET with florbetapir compared with neuropathology at autopsy for detection of neuritic amyloid-beta plaques: a prospective cohort study. Lancet Neurol. 2012;11:669-78.

35. Joshi AD, Pontecorvo MJ, Clark CM, Carpenter AP, Jennings DL, Sadowsky $\mathrm{CH}$, et al. Performance characteristics of amyloid PET with florbetapir $\mathrm{F} 18$ in patients with Alzheimer's disease and cognitively normal subjects. J Nucl Med. 2012;53:378-84.

36. Liu E, Wang D, Sperling R, Salloway S, Fox NC, Blennow K, et al. Biomarker pattern of ARIA-E participants in phase 3 randomized clinical trials with bapineuzumab. Neurology. 2018;90:e877-e86.

37. Schmidt ME, Chiao P, Klein G, Matthews D, Thurfjell L, Cole PE, et al. The influence of biological and technical factors on quantitative analysis of amyloid PET: points to consider and recommendations for controlling variability in longitudinal data. Alzheimers Dement. 2015;11:1050-68.

38. Klein G, Delmar P, Abi-Saab D, Andjelkovic M, Milosavljevic-Ristic S, Seibyl J, et al. Methodologic considerations for calculating standard uptake value ratio amyloid reduction in the gantenerumab open label extension studies. Human Amyloid Imaging (HAI) Conference; 2018; Miami, FL, USA.

39. von Rosenstiel P, Gheuens S, Chen T, O'Gorman J, Chiao P, Wang G, et al. Aducanumab titration dosing regimen: 24-month analysis from PRIME, a randomized, double-blind, placebo-controlled phase $1 \mathrm{~b}$ study in patients with prodromal or mild Alzheimer's disease (\$2.003). Neurology. 2018;90(15 Supplement):S2 003.

40. Swanson C, Zhang Y, Dhadda S, Wang J, Kaplow J, Lai RYK, et al. Treatment of early AD subjects with BAN2401, an anti-A 3 protofibril monoclonal antibody, significantly clears amyloid plaque and reduces clinical decline. Alzheimers Dement. 2018;14(Suppl):1668.
41. Fleisher AS, Lowe SL, Liu P, Shcherbinin S, Li L, Chua L, et al. Safety, tolerability, pharmacokinetics, and pharmacodynamics of single and multiple intravenous doses of LY3002813, a $\beta$-amyloid plaque-specific antibody, in patients iwth mild cognitive impairment due to Alzheimer's Disease or mild to moderate Alzheimer's Dementia. Alzheimer's Association International Conference (AAIC); 2018; Chicago.

42. Brody M, Liu E, Di J, Lu M, Margolin RA, Werth JL, et al. A phase II, randomized, double-blind, placebo-controlled study of safety, pharmacokinetics, and biomarker results of subcutaneous bapineuzumab in patients with mild to moderate Alzheimer's disease. J Alzheimers Dis. 2016; 54:1509-19.

43. Siemers ER, Sundell KL, Carlson C, Case M, Sethuraman G, Liu-Seifert H, et al. Phase 3 solanezumab trials: secondary outcomes in mild Alzheimer's disease patients. Alzheimers Dement. 2016;12:110-20.

44. Kennedy ME, Stamford AW, Chen X, Cox K, Cumming JN, Dockendorf MF, et al. The BACE1 inhibitor verubecestat (MK-8931) reduces CNS betaamyloid in animal models and in Alzheimer's disease patients. Sci Transl Med. 2016:8:363ra150.

45. Villemagne VL, Rowe CC, Barnham KJ, Cherny R, Woodward M, Bozinosvski $\mathrm{S}$, et al. A randomized, exploratory molecular imaging study targeting amyloid beta with a novel 8-OH quinoline in Alzheimer's disease: the PBT2204 IMAGINE study. Alzheimers Dement (N Y). 2017;3:622-35.

46. Cselenyi Z, Farde L. Quantification of blood flow-dependent component in estimates of beta-amyloid load obtained using quasi-steady-state standardized uptake value ratio. J Cereb Blood Flow Metab. 2015;35:1485-93.

47. Ottoy J, Verhaeghe J, Niemantsverdriet E, Engelborghs S, Stroobants S, Staelens S. A simulation study on the impact of the blood flow-dependent component in [18F]AV45 SUVR in Alzheimer's disease. PLoS One. 2017;12: e0189155.

48. van Berckel BN, Ossenkoppele R, Tolboom N, Yaqub M, Foster-Dingley JC, Windhorst $A D$, et al. Longitudinal amyloid imaging using 11C-PiB: methodologic considerations. J Nucl Med. 2013;54:1570-6.

49. Egan MF, Kost J, Tariot PN, Aisen PS, Cummings JL, Vellas B, et al. Randomized trial of verubecestat for mild-to-moderate Alzheimer's disease. N Engl J Med. 2018;378:1691-703.

50. Fleisher AS, Lowe SL, Liu P, Shcherbinin S, Li L, Chua L, et al. Significant and sustained florbetapir F18 uptake reduction in patients with symptomatic Alzheimer's disease with LY3002813, a $\beta$-amyloid plaque-specific antibody. Alzheimers Dement. 2018;14(Suppl):239.

51. Su Y, Blazey TM, Snyder AZ, Raichle ME, Marcus DS, Ances BM, et al. Partial volume correction in quantitative amyloid imaging. Neuroimage. 2015;107: $55-64$.

52. Thomas BA, Erlandsson K, Modat M, Thurfjell L, Vandenberghe R, Ourselin S, et al. The importance of appropriate partial volume correction for PET quantification in Alzheimer's disease. Eur J Nucl Med Mol Imaging. 2011;38:1104-19.

53. Schwarz CG, Gunter JL, Lowe VJ, Weigand S, Vemuri P, Senjem ML, et al. A comparison of partial volume correction techniques for measuring change in serial amyloid PET SUVR. J Alzheimers Dis. 2019;67:181-95.

54. Biogen. Biogen and Eisai to discontinue Phase 3 ENGAGE and EMERGE trials of aducanumab in Alzheimer's disease [news release]. 21 March 2019. http://investors.biogen.com/news-releases/news-release-details/biogen-andeisai-discontinue-phase-3-engage-and-emerge-trials. Accessed 4 Nov 2019.

55. Biogen. Biogen plans regulatory filing for aducanumab in Alzheimer's disease based on new analysis of larger dataset from phase 3 studies. https://investors. biogen.com/news-releases/news-release-details/biogen-plans-regulatory-filingaducanumab-alzheimers-disease. Accessed 4 Nov 2019.

56. Abi-Saab D, Andjelkovic M, Pross N, Delmar P, Voyle N, Klein G, et al. Update on the safety and tolerability of gantenerumab in the ongoing open-label extension (OLE) of the Marguerite RoAD study in patients with prodromal Alzheimer's disease (AD) after approximately 2 years of study duration. Alzheimers Dement. 2018;14(Suppl):P241 01-09-04.

57. Andjelkovic M, Abi-Saab D, Delmar P, Pross N, Voyle N, Klein G, et al. Update on the safety and tolerability of gantenerumab in the ongoing open-label extension of the SCarlet RoAD study in patients with prodromal Alzheimer's disease after approximately 2 years of study duration. Alzheimers Dement. 2018;14(Suppl):P241 01-09-05.

\section{Publisher's Note}

Springer Nature remains neutral with regard to jurisdictional claims in published maps and institutional affiliations. 